\title{
Comparison of Direct Eulerian Godunov and Lagrange Plus Remap, Artificial Viscosity Schemes
}

R. B. Pember, R. W. Anderson

This article was submitted to $15^{\text {th }}$ American Institute of Aeronautics and Astronautics Computational Fluid Dynamics Conference, Anaheim, CA, U.S. Department of Energy June 11-14, 2001

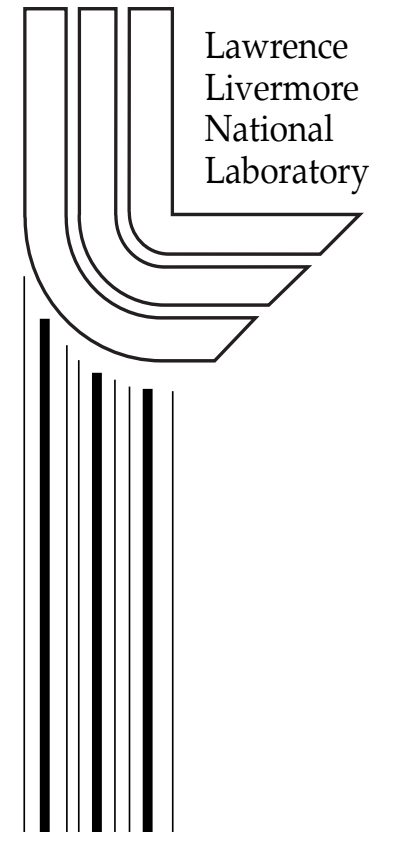

March 30, 2001 


\section{DISCLAIMER}

This document was prepared as an account of work sponsored by an agency of the United States Government. Neither the United States Government nor the University of California nor any of their employees, makes any warranty, express or implied, or assumes any legal liability or responsibility for the accuracy, completeness, or usefulness of any information, apparatus, product, or process disclosed, or represents that its use would not infringe privately owned rights. Reference herein to any specific commercial product, process, or service by trade name, trademark, manufacturer, or otherwise, does not necessarily constitute or imply its endorsement, recommendation, or favoring by the United States Government or the University of California. The views and opinions of authors expressed herein do not necessarily state or reflect those of the United States Government or the University of California, and shall not be used for advertising or product endorsement purposes.

This is a preprint of a paper intended for publication in a journal or proceedings. Since changes may be made before publication, this preprint is made available with the understanding that it will not be cited or reproduced without the permission of the author.

This work was performed under the auspices of the United States Department of Energy by the University of California, Lawrence Livermore National Laboratory under contract No. W-7405-Eng-48.

This report has been reproduced directly from the best available copy.

Available electronically at http://www.doc.gov/bridge

Available for a processing fee to U.S. Department of Energy

And its contractors in paper from

U.S. Department of Energy

Office of Scientific and Technical Information

P.O. Box 62

Oak Ridge, TN 37831-0062

Telephone: (865) 576-8401

Facsimile: (865) 576-5728

E-mail: reports@adonis.osti.gov

Available for the sale to the public from

U.S. Department of Commerce

National Technical Information Service

5285 Port Royal Road

Springfield, VA 22161

Telephone: (800) 553-6847

Facsimile: (703) 605-6900

E-mail: orders@ntis.fedworld.gov

Online ordering: http://www.ntis.gov/ordering.htm

OR

Lawrence Livermore National Laboratory

Technical Information Department's Digital Library

http://www.llnl.gov/tid/Library.html 
AIAA 2001-2644

\title{
COMPARISON OF DIRECT EULERIAN GODUNOV AND LAGRANGE PLUS REMAP ARTIFICIAL VISCOSITY SCHEMES
}

\author{
Richard B. Pember* and Robert W. Anderson ${ }^{\dagger}$ \\ Center for Applied Scientific Computing \\ Lawrence Livermore National Laboratory, Livermore, CA 94550
}

\begin{abstract}
$\underline{\text { Abstract }}$
We compare two algorithms for solving the equations of unsteady inviscid compressible flow in an Eulerian frame: a staggered grid, Lagrange plus remap artificial viscosity scheme and a cell-centered, direct Eulerian higher-order Godunov scheme. We use the two methods to compute solutions to a number of one- and two-dimensional problems. Our results show the accuracy of the two schemes to be generally equivalent. In a 1984 survey paper by Woodward and Colella, the Lagrange plus remap approach did not compare favorably with the higher-order Godunov methodology. We examine, therefore, how certain features of the staggered grid scheme considered here contribute to its improved accuracy. The critical features are shown to be the use of a monotonic artificial viscosity in the Lagrange step and, in the remap step, the use of a corner transport upwind scheme with van Leer limiters in conjunction with separate advection of internal and kinetic energies.
\end{abstract}

\section{Introduction}

Shock capturing methods have always played a central role in the computational modeling of compressible flows. Consequently there has always been interest in comparing the various methods. Seventeen years ago there appeared one such comparison by Woodward and Colella. ${ }^{1}$ The methods examined therein include a direct Eulerian version of the piecewise-parabolic method (PPM) and two different artificial viscosity schemes: MacCormack's scheme, a cell-centered method based on the LaxWendroff scheme, and BBC, a staggered grid, Lagrange plus remap scheme with a von NeumannRichtmyer artificial viscosity. The article includes a ranking of the accuracy of the schemes in which PPM is ranked first, BBC fourth, and MacCormack's scheme fifth. Although it was not the intent

\footnotetext{
* Staff Scientist, AIAA member.

$\dagger$ Postdoctoral researcher, AIAA member.

Copyright (C) 2001 by the American Institute of Aeronautics and Astronautics, Inc. All rights reserved.
}

of the authors, the reader is left with an impression of the superiority of higher-order upwind methods and the inferiority of artificial viscosity schemes. (We note that the representative linear hybridization code, ETBFCT, was ranked third. The present paper discusses neither the linear hybridization approach nor flux-corrected transport (FCT) schemes.)

In the intervening years, direct Eulerian methods using an artificial viscosity have certainly demonstrated comparable accuracy with higher-order upwind schemes. This success has been accomplished in part by the use of linear hybridization to formulate the artificial viscosity terms. ${ }^{2}$ The approach represented by $\mathrm{BBC}$, however, is generally no longer considered viable for Eulerian calculations. In particular, one finds interpretations of the conclusions of Woodward and Colella that the Lagrange plus remap, artificial viscosity approach is inherently less accurate than the direct Eulerian higher-order Godunov methodology. ${ }^{3,4}$

The staggered grid, artificial viscosity Lagrange plus remap approach does remain important, however, in the context of arbitrary Lagrangian-Eulerian (ALE) methods. ${ }^{5-9}$ An operator split ALE method for compressible flow typically consists of three steps: a Lagrange step, in which the equations of gas dynamics are solved on a grid that moves with the fluid velocity; a grid motion step in which the Lagrangian grid is modified, if necessary, to reduce grid distortion; and a remap step in which the solution at the end of the Lagrangian step is conservatively interpolated onto the modified grid via an advection scheme.

Motivated by its importance in the context of split ALE schemes, in this paper we reexamine the comparison of the staggered grid, artificial viscosity Lagrange plus remap approach with the direct $\mathrm{Eu}-$ lerian, higher-order Godunov methodology. We first formulate and discuss the staggered grid Lagrange plus remap scheme used in our comparison. The Lagrange step follows a standard approach, namely, the scheme due to Tipton ${ }^{10}$ which is itself based on HEMP. ${ }^{11,12}$ The remap step uses a variation of the 
corner transport upwind method of Colella ${ }^{13}$ as formulated for moving quadrilateral grids. ${ }^{14}$ (To our knowledge, only Jun ${ }^{15}$ has applied this scheme in a remap step as well.) The remap step includes an approach standard to staggered grid ALE schemes, ${ }^{16}$ namely, a total energy advection scheme in which kinetic and internal energy are advected separately to determine the remapped total energy. This approach, while still total energy conserving, overcomes the difficulties reported by Woodward and Colella ${ }^{1}$ in the solution of the interacting blast wave problem; specifically we are able to use van Leer limiters while avoiding early time overshoots in density.

The second method in our comparison is the higher-order Godunov scheme due to Colella. ${ }^{4,17,18}$ After briefly reviewing this scheme, we use both methods to compute solutions to a number of oneand two-dimensional problems. (We will refer to the two methods as the Lagrange plus remap scheme and the higher-order Godunov scheme.) The test problems include two of the those examined by Woodward and Colella, ${ }^{1}$ the interacting blast wave and the double Mach reflection of a strong shock, as well as a shock refraction and the LeBlanc shock tube. Our results demonstrate that the accuracy of the two schemes is generally equivalent, each scheme displaying both relative strengths and weaknesses. The results show in particular that the improved accuracy of the present Lagrange plus remap scheme relative to $\mathrm{BBC}$ is due to several key features of the scheme: a monotonic artificial viscosity in the Lagrange step and the use of the corner transport upwind scheme in the remap step with van Leer limiters in conjunction with separate advection of internal and kinetic energies.

\section{Governing equations}

The equations of single fluid gas dynamics can be expressed in either of two equivalent formulations, the conservative and the material derivative forms. In two dimensional rectangular coordinates, the conservative formulation is

$$
\frac{\partial U}{\partial t}+\frac{\partial F(U)}{\partial x}+\frac{\partial G(U)}{\partial y}=0
$$

where

$U=(\rho, \rho u, \rho v, \rho E)^{T}$,

$F(U)=\left(\rho u, \rho u^{2}+p, \rho u v, \rho u E+p u\right)^{T}$,

$G(U)=\left(\rho v, \rho u v, \rho v^{2}+p, \rho v E+p v\right)^{T}$,

and $E=1 / 2\left(u^{2}+v^{2}\right)+e$, while the material derivative formulation is

$$
\frac{D \rho}{D t}=-\rho \nabla \cdot U
$$

$$
\begin{aligned}
\rho \frac{D U}{D t} & =-\nabla p \\
\frac{D e}{D t} & =-\frac{p}{\rho} \nabla \cdot U=-\frac{D}{D t}\left(\frac{1}{\rho}\right) p
\end{aligned}
$$

where $U=(u, v)^{T}$. For all examples considered in this paper, $p$ is given by the polytropic equation of state $p=p(\rho, e)=(\gamma-1) \rho e$.

\section{Lagrange Plus Remap Scheme}

The Lagrange plus remap scheme is an operator split scheme consisting of two steps: a Lagrange step, in which the equations of gas dynamics are advanced to the new time on a grid that moves with the fluid; and the remap step, in which the solution is conservatively interpolated onto the original grid via an advection over a pseudo-timestep.

We solve the equations on a structured grid of quadrilateral cells. The flow variables are staggered: $\rho$ and $e$ are cell-centered quantities while $U=(u, v)$ is node-centered. The grid itself is defined by the positions $X=(x, y)$ of the nodes. We use the following indexing convention. The cells themselves are indexed as $i j$, the four nodes as $i \pm 1 / 2, j \pm 1 / 2$, and the four bounding faces as $i \pm 1 / 2, j$ and $i, j \pm 1 / 2$.

The Lagrange step uses a predictor-corrector formulation in which the flow variables are not staggered in time and which otherwise follows the scheme due to Tipton. ${ }^{10}$ The scheme uses a multidimensional form ${ }^{12}$ of the scalar monotonic artificial viscosity due to Christensen. ${ }^{19}$ The artificial viscosity $q$ is a cell-centered quantity that acts in the same manner as pressure. In one space dimension, this viscosity is given by

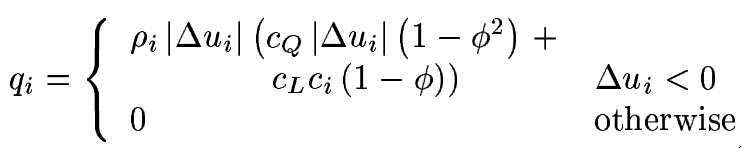

where $\Delta u_{i}=u_{i+1 / 2}-u_{i-1 / 2}, c$ is the sound speed, and

$$
\begin{aligned}
\phi_{i}= & \max \left(0, \min \left(1,2 R_{i+1}, 2 R_{i-1},\right.\right. \\
& \left.\left..5\left(R_{i+1}+R_{i-1}\right)\right)\right),
\end{aligned}
$$

where $R_{i \pm 1}=\Delta u_{i \pm 1} / \Delta u_{i}$. When $\phi=0$, the viscosity reduces to what is often called the standard Lagrange viscosity. ${ }^{20} c_{Q}$ and $c_{L}$ are constants that are generally set to $.25(\gamma+1)$ and .5 for polytropic gases. ${ }^{6}$ The effect of the limiters $1-\phi$ and $1-\phi^{2}$ is to reduce the viscosity in regions with moderate velocity gradients and thereby reduce the numerical diffusion of the scheme. 
The Lagrange step uses the HEMP ${ }^{11,12}$ spatial discretization of the momentum equation. Figure 1 shows four computational cells - A, B,C,D - and the nine nodes associated with them. The mass of node 0 is defined by

$$
m_{0}=\left(\rho_{A} V_{A}+\rho_{B} V_{B}+\rho_{C} V_{C}+\rho_{D} V_{D}\right) / 2
$$

where $V$ is the cell volume. By the divergence theorem,

$$
\int_{\overline{1357}} \nabla(p+q) d V=\int_{\partial(\overline{1357})}(p+q) \boldsymbol{n d s},
$$

where $\boldsymbol{n}$ is the outward facing normal, and $d V$ and $d s$ the standard area and length measures. (The notation $\overline{p_{1} p_{2} \ldots p_{n}}$ is used to denote the $n$-gon with vertices $p_{1}, p_{2}, \ldots, p_{n}$.) The semi-discrete form of the velocity update is then

$$
\frac{D U}{D t} \equiv a=\frac{1}{m_{0}} \int_{\partial(\overline{1357)}}(p+q) \boldsymbol{n d s} ;
$$

the integral is approximated using a constant pressure in each cell. Note that this discretization is conservative: since $\int \boldsymbol{n} d s=0$ over a closed curve, for constant pressure elements

$$
\int_{\partial(\overline{a b c d e f g h})}(p+q) \boldsymbol{n d} d s=1 / 2 \int_{\partial(\overline{1357})}(p+q) \boldsymbol{n} d s .
$$

(Note that an hourglass filter ${ }^{12}$ is not used in the current scheme because the Lagrangian solution is always remapped to the original grid.)

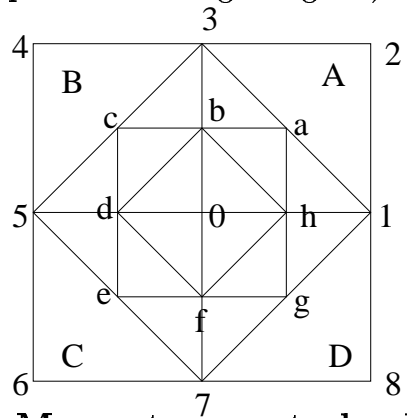

Fig. 1 Momentum control volume.

A predictor-corrector scheme is used to solve equations (2), (4), and (6). In the predictor, we compute predicted values of $\rho, U, e, X$, and $V$ at time $n+1$. In the corrector, we average the predicted time $n+1$ values with the time $n$ values to form time-centered source terms. The predictor-corrector has the following steps:

1. Compute $a^{n}$ using time $n$ values and equation (6).

2. Compute the predicted velocity:

$$
U_{i+1 / 2, j+1 / 2}^{n+1}=U_{i+1 / 2, j+1 / 2}^{n}+\Delta t a_{i+1 / 2, j+1 / 2}^{n} .
$$

3. Compute the predicted node positions:

$$
\begin{aligned}
X_{i+1 / 2, j+1 / 2}^{n+1}= & X_{i+1 / 2, j+1 / 2}^{n}+ \\
& .5 \Delta t\left(U_{i+1 / 2, j+1 / 2}^{n}+U_{i+1 / 2, j+1 / 2}^{n+1}\right) .
\end{aligned}
$$

4. Compute the predicted zone volumes $V_{i j}^{n+1}$.

5. Compute the predicted densities:

$$
\rho_{i j}^{n+1}=\rho_{i j}^{n} V_{i j}^{n} / V_{i j}^{n+1} .
$$

6. Compute the predicted internal energy

$$
e_{i j}^{n+1}=e_{i j}^{n}-\left(p_{i j}^{n}+q_{i j}^{n}\right)\left(1 / \rho_{i j}^{n+1}-1 / \rho_{i j}^{n}\right) .
$$

7. Compute $a^{n+1}$ predicted time $n+1$ values and equation (6).

8. Repeat steps 2-6 using time centered values of $a, p$, and $q$.

Note that in step 3 we use a time-centered velocity even in the predictor step. This ensures that the restriction of the scheme to one dimension conserves total energy. ${ }^{21}$

The Lagrange step has a time step restriction of $\Delta t_{L a g}^{n}=\sigma \min _{i j} l_{i j} / c_{i j}^{n}$, where $l_{i j}$ is a measure of the shortest distance across cell $i j$ and the CourantFriedrichs-Lewy (CFL) number $\sigma$ is a positive constant, $\sigma \leq 1$.

In the remap step we use an advection scheme to conservatively interpolate the solution obtained above from the grid defined by $X^{n+1}$ onto the original grid defined by $X^{n}$. This interpolation is formulated as an initial value problem in which

$$
\partial w / \partial \tau=0
$$

is solved on a moving grid. $w$ and $\tau$ denote an arbitrary scalar quantity defined on the grid and a pseudo-time, respectively. At $\tau=0, w$ is defined by its value at the end of the Lagrange step. We integrate (7) from $\tau=0$ to $\tau=\tau_{f}$. Over this time, the grid moves from $\boldsymbol{x}^{\text {old }}=X^{n+1}$ to $\boldsymbol{x}^{\text {new }}=X^{n}$. (The superscripts "old" and "new" denote pre- and post-remap values, respectively.) The grid velocity is defined by $\boldsymbol{s}=\left(\boldsymbol{x}^{\text {new }}-\boldsymbol{x}^{\text {old }}\right) / \tau_{f}$. We now transform (7) to index space coordinates $(\xi, \eta)=(i, j)$ and thereby obtain the equation

$$
\frac{\partial J w}{\partial \tau}-\frac{\partial}{\partial \xi}\left(\boldsymbol{n}^{\xi} \cdot \boldsymbol{s} w\right)-\frac{\partial}{\partial \eta}\left(\boldsymbol{n}^{\eta} \cdot \boldsymbol{s} w\right)=0
$$

where

$J=x_{\xi} y_{\eta}-y_{\xi} x_{\eta}, \boldsymbol{n}^{\xi}=\left(y_{\eta},-x_{\eta}\right), \boldsymbol{n}^{\eta}=\left(-y_{\xi}, x_{\xi}\right)$.

We now solve (8) with a conservative difference scheme. 
In order to ensure freestream preservation the fluxes used in the conservative difference scheme must be formulated as products of edge values and either transport volumes (in the case of density) or transport masses (in the case of all other quantities). ${ }^{22}$ The transport volume associated with an edge is the signed quadrilateral formed by the old and new edges, and the segments connecting the old and new node positions. In Figure 2, the arrows point from the old grid to the new grid. A, B, G, and $\mathrm{H}$ represent positive transport volumes while $\mathrm{C}$, $\mathrm{D}, \mathrm{E}$, and $\mathrm{F}$ represent negative ones. More precisely, the transport volumes $\delta \sigma$ are defined at $\xi$-edges by

$$
\begin{aligned}
\delta \sigma_{i+1 / 2, j}=.5( & \left(x_{i+1 / 2, j+1 / 2}^{\text {old }}-x_{i+1 / 2, j-1 / 2}^{\text {new }}\right) \\
& \left(y_{i+1 / 2, j-1 / 2}^{\text {old }}-y_{i+1 / 2, j+1 / 2}^{n e w}\right)- \\
& \left(x_{i+1 / 2, j-1 / 2}^{\text {old }}-x_{i+1 / 2, j+1 / 2}^{\text {new }}\right) \\
& \left.\left(y_{i+1 / 2, j+1 / 2}^{\text {old }}-y_{i+1 / 2, j-1 / 2}^{\text {new }}\right)\right)
\end{aligned}
$$

with a similar expression for the $\eta$-edges. Volume fluxes are then defined by $F_{i+1 / 2, j}=-\delta \sigma_{i+1 / 2, j}$ and $F_{i, j+1 / 2}=-\delta \sigma_{i, j+1 / 2}$, i.e.,

$V_{i j}^{\text {new }}=V_{i j}^{\text {old }}+\left(F_{i-1 / 2, j}-F_{i+1 / 2, j}\right)+\left(F_{i, j-1 / 2}-F_{i, j+1 / 2}\right)$.

Transport masses for cell-centered quantities can be defined by the product of the density and the transport volume at an edge:

$$
\begin{aligned}
& \delta m_{i+1 / 2, j}=\rho_{i+1 / 2, j} \delta \sigma_{i+1 / 2, j} \\
& \delta m_{i, j+1 / 2}=\rho_{i, j+1 / 2} \delta \sigma_{i, j+1 / 2} .
\end{aligned}
$$

Mass fluxes are defined by $F_{i+1 / 2, j}=-\delta m_{i+1 / 2, j}$ and $F_{i, j+1 / 2}=-\delta m_{i, j+1 / 2}$, i.e.,

$m_{i j}^{\text {new }}=m_{i j}^{\text {old }}+\left(F_{i-1 / 2, j}-F_{i+1 / 2, j}\right)+\left(F_{i, j-1 / 2}-F_{i, j+1 / 2}\right)$,

where $m_{i j}=\rho_{i j} V_{i j}$ is the mass of cell $i j$.

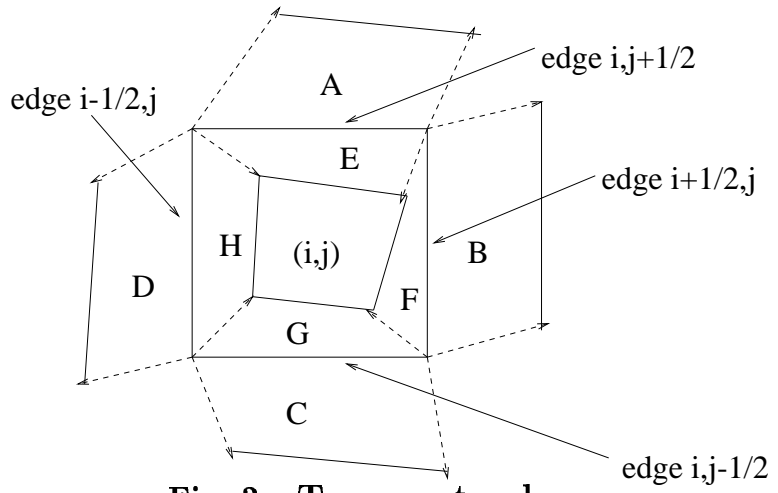

Fig. 2 Transport volumes.

The control volume for momentum remap is considered a logical rectangle; for node 0 in Figure
1 , the control volume is aceg. The four bounding faces of the control volume associated with the node at $(i+1 / 2, j+1 / 2)$ are indexed as $(i, j+1 / 2)$, $(i+1, j+1 / 2),(i+1 / 2, j)$, and $(i+1 / 2, j+1)$. The transport masses for the $\xi$-faces are given by

$$
\begin{aligned}
\delta m_{i+1 / 2, j}^{\text {nodal }}= & 1 / 4\left(\delta m_{i, j+1 / 2}+\delta m_{i+1, j+1 / 2}\right. \\
& \left.+\delta m_{i, j-1 / 2}+\delta m_{i+1, j-1 / 2}\right)
\end{aligned}
$$

with a similar expression for the $\eta$-faces. The transport mass must be defined in this manner to ensure freestream preservation of velocity given the HEMP discretization of the momentum equation.

The remap algorithm can now be summarized as follows:

1. Compute transport volumes at all edges.

2. Compute densities at all edges.

3. Compute transport masses using (9) and (10).

4. Remap density by

$$
\begin{aligned}
V_{i j}^{\text {new }} \rho_{i j}^{\text {new }}= & V_{i j}^{\text {old }} \rho_{i j}^{\text {old }}+\left(F_{i-1 / 2, j}-F_{i+1 / 2, j}\right) \\
& +\left(F_{i, j-1 / 2}-F_{i, j+1 / 2}\right)
\end{aligned}
$$

where

$$
\begin{aligned}
& F_{i+1 / 2, j}=-\rho_{i+1 / 2, j} \delta \sigma_{i+1 / 2, j} \\
& F_{i, j+1 / 2}=-\rho_{i, j+1 / 2} \delta \sigma_{i, j+1 / 2} .
\end{aligned}
$$

5. Remap $w=u, v$ by

$$
\begin{aligned}
& m_{i+1 / 2, j+1 / 2}^{\text {new }} w_{i+1 / 2, j+1 / 2}^{\text {new }}=m_{i+1 / 2, j+1 / 2}^{\text {old }} w_{i+1 / 2, j+1 / 2}^{\text {old }}+ \\
& \left(F_{i, j+1 / 2}-F_{i+1, j+1 / 2}\right)+\left(F_{i+1 / 2, j}-F_{i+1 / 2, j+1}\right)
\end{aligned}
$$

where

$$
\begin{aligned}
& F_{i, j+1 / 2}=-w_{i, j+1 / 2} \delta m_{i, j+1 / 2}^{\text {nodal }} \\
& F_{i+1 / 2, j}=-w_{i+1 / 2, j} \delta m_{i+1 / 2, j}^{\text {nodal }} .
\end{aligned}
$$

6. Remap $w=k e, e$ by

$$
\begin{aligned}
m_{i j}^{\text {new }} w_{i j}^{\text {new }}= & m_{i j}^{\text {old }} w_{i j}^{\text {old }}+\left(F_{i-1 / 2, j}-F_{i+1 / 2, j}\right)+ \\
& \left(F_{i, j-1 / 2}-F_{i, j+1 / 2}\right)
\end{aligned}
$$

where

$$
\begin{aligned}
F_{i+1 / 2, j} & =-w_{i+1 / 2, j} \delta m_{i+1 / 2, j} \\
F_{i, j+1 / 2} & =-w_{i, j+1 / 2} \delta m_{i, j+1 / 2}, \text { and } \\
k e_{i j}^{\text {old }} & =1 / 4 \sum_{\text {nodes of } i j} 1 / 2\left(u^{2}+v^{2}\right)^{\text {old }} .
\end{aligned}
$$

7. Enforce conservation of total energy by

$$
\begin{aligned}
e_{i j}^{n e w}= & e_{i j}^{n e w}+k e_{i j}^{n e w}- \\
1 / 4 & \sum_{\text {nodes of } i j} 1 / 2\left(u^{2}+v^{2}\right)^{n e w} .
\end{aligned}
$$


The remap algorithm described above is independent of the specific manner in which edge values are computed. We use the corner transport upwind scheme due to Colella ${ }^{13}$ as formulated for moving quadrilateral grids by Bell et $a .^{14}$ for the computation of edge values. The algorithm applies to both cell-centered and node-centered control volumes. The algorithm has two basic steps. First, left and right (top and bottom) states are determined at each $\xi_{-}\left(\eta_{-}\right)$edge by a first-order Taylor expansions in space and time. A unique edge value is then determined by upwinding with either $-\delta \sigma$ (density) or $-\delta m$ (other quantities): if $-\delta \sigma$ or $-\delta m$ is positive, the left or bottom state is chosen; otherwise, the right or top state is chosen.

To perform the Taylor expansion we first compute compute van Leer limited ${ }^{23}$ central differences $\Delta_{\xi} w$, $\Delta_{\eta} w$ in $\xi$ - and $\eta$ - directions. In the $\xi$ - direction, these are computed by

$$
\begin{aligned}
\Delta_{\xi} w_{i j}= & \min \left(\left|\Delta^{C} w\right|, 2\left|\Delta^{L} w\right|, 2\left|\Delta^{R} w\right|\right) \\
& \quad \times \operatorname{sgn}\left(\Delta^{C} w\right) \text { if } \Delta^{L} w \Delta^{R} w>0 \\
= & 0, \text { otherwise, }
\end{aligned}
$$

where $\Delta^{L} w=w_{i}-w_{i-1}, \Delta^{R} w=w_{i+1}-w_{i}$, and $\Delta^{C} w=1 / 2\left(w_{i+1}-w_{i-1}\right)$. The states on the left sides of $\xi$ - edges are defined by

$$
w_{i+1 / 2, j, L}^{n+1 / 2}=\left(w+\frac{\Delta \xi}{2} w_{\xi}+\frac{\Delta \tau}{2} w_{t}\right)_{i j}^{n}
$$

Similar expressions can be obtained for the right sides of $\xi$ - edges and the top and bottom sides of $\eta$ - edges. Using (8) we can express $w_{t}$ in terms of spatial derivatives and obtain

$$
\begin{aligned}
w_{i+1 / 2, j, L}^{n+1 / 2}= & \left(w+\left(\frac{\Delta \xi}{2}+\frac{\Delta \tau n^{\xi} \cdot s}{2 J}\right) w_{\xi}+\right. \\
& \left.\frac{\Delta \tau n^{\eta} \cdot s}{2 J} w_{\eta}\right)_{i j}^{n}
\end{aligned}
$$

We evaluate $w_{\xi}$ as $\Delta_{\xi} w / \Delta \xi$ and $w_{\eta}$ as $\left(w_{i, j+1 / 2}^{p}-\right.$ $\left.w_{i, j-1 / 2}^{p}\right) / \Delta \eta$. In the latter expression $w_{i, j+1 / 2}^{p}$ is determined by performing one-dimensional Taylor expansions in the $\eta$ direction, i.e.,

$$
\begin{gathered}
w_{i, j+1 / 2, B}^{p}=\left(w+\left(\frac{\Delta \eta}{2}+\frac{\Delta \tau \boldsymbol{n}^{\eta} \cdot \boldsymbol{s}}{2 J}\right) w_{\eta}\right)_{i j}^{n} \\
w_{i, j+1 / 2, T}^{p}=\left(w-\left(\frac{\Delta \eta}{2}-\frac{\Delta \tau \boldsymbol{n}^{\eta} \cdot \boldsymbol{s}}{2 J}\right) w_{\eta}\right)_{i+1, j}^{n},
\end{gathered}
$$

and upwinding the resultant top and bottom edge states.
Although there is no time step associated with the remap step, there is a time step restriction of $\Delta t_{\text {remap }}^{n}=\sigma \min _{i, j} l_{i j} / \max \left|U_{i \pm 1 / 2, j \pm 1 / 2}^{n}\right|, \quad \sigma \leq 1$, which is equivalent to the restriction that each cell at the end of the Lagrange step have some intersection with itself in the original grid. The time step used by the full Lagrange plus remap scheme is $\Delta t^{n}=\min \left(\Delta t_{L a g}^{n}, \Delta t_{\text {remap }}^{n}\right)$.

\section{Higher-order Godunov Scheme}

The higher-order Godunov method is a spatially split version of the scheme due to Colella. ${ }^{4,17,18}$ We use a uniform computational grid with cell widths $\Delta x, \Delta y$ indexed by $i, j$. The bounding faces of cell $i, j$ are indexed as $i \pm 1 / 2, j$ and $i, j \pm 1 / 2$. The flow variables $\rho, u, v$, and $E$ are all cell-centered. In the $x$-sweep (the $y$-sweep is similar), we solve

$$
\frac{\partial U}{\partial t}+\frac{\partial F(U)}{\partial x}=0
$$

In formulating the $x$-sweep, the following quasilinear, non-conservative form of (12) is used:

$$
\frac{\partial q}{\partial t}+A(q) \frac{\partial q}{\partial x}=0
$$

where $q=\left(q_{1}, \ldots, q_{5}\right)^{T}=(\rho, u, v, p, \rho e)^{T}$

$$
A(q)=\left(\begin{array}{ccccc}
u & \rho & 0 & 0 & 0 \\
0 & u & 0 & 1 / \rho & 0 \\
0 & 0 & u & 0 & 0 \\
0 & \rho c^{2} & 0 & u & 0 \\
0 & \rho h & 0 & 0 & u
\end{array}\right)
$$

and $h=e+p / \rho$ is the specific enthalpy. The primitive variables are thermodynamically redundant so that only one equation of state evaluation per sweep is required.

The algorithm to integrate (12) has three basic steps. For simplicity, we suppress the index $j$ and let $U^{n+1}$ denote the value of $U$ at the end of the $x$-sweep:

1. Compute time-centered left and right states, $q_{i+1 / 2, L}^{n+1 / 2}$ and $q_{i+1 / 2, R}^{n+1 / 2}$, at each $x$-cell face.

2 . Solve the Riemann problem at each cell face with the left and right states computed in (2) and evaluate that solution along the ray $x / t=0$ to obtain $q_{i+1 / 2}^{n+1 / 2}$.

3. Compute $U_{i}^{n+1}$ by conservative differencing.

The first step basically follows the algorithm described by Miller and Puckett ${ }^{4}$ applied to the quasilinear system (13). Fourth-order spatial slopes ${ }^{24}$ and a slope flattening technique ${ }^{25}$ are used here. 
The second step uses a linearized approximate Riemann solver with a special treatment for rarefactions $^{26}$ to solve the Riemann problems defined in step 1. The last step uses the Riemann problem solutions to define numerical fluxes and update $U$ conservatively. In regions of convergent flow, an explicit diffusive flux ${ }^{25}$ is added to the numerical flux in order that a small amount of additional numerical dissipation be present in the vicinity of shocks. The $x$-sweep has a time step restriction of $\Delta t^{n}=\sigma \min _{i, j} \Delta x /\left(\left|u_{i j}^{n}\right|+c_{i j}^{n}\right), \sigma \leq 1$.

\section{Numerical results}

For all problems, we use a uniform grid of square cells. The CFL number is .9 unless noted otherwise.

\section{Interacting blast wave.}

The flow domain for this one-dimensional problem ${ }^{1,19}$ has length one with reflecting walls at both end. The gas is polytropic with $\gamma=1.4$. At $t=0$ the gas is at rest with density 1 . The initial pressure is 1000 in the leftmost tenth of the domain, 100 in the rightmost tenth, and .01 elsewhere. Initially, two shock waves and two contact discontinuities develop at the initial discontinuities and propagate towards one another, while two rarefactions develop, propagate towards the walls, and reflect off them. As time progresses, these six initial waves interact and create additional contact discontinuities.

Figure 3 displays the density computed by the higher-order Godunov and the Lagrange plus remap schemes at $t=.038$ on a 1200 zone domain. To obtain a baseline solution, we also compute the flow both with a Lagrangian higher-order Godunov method $^{27}$ and with the staggered grid Lagrange scheme on 3600 zone domains. The initial zoning for both Lagrangian computations is spatially uniform. The densities at $t=.038$ for all four computations are displayed in Figure 3. The two Lagrangian solutions are virtually indistinguishable and therefore serve as a baseline solution. We note that these solutions themselves have a flaw, a spurious overshoot at $x \approx .765$. The magnitude of the overshoot is the only discernible difference between the two sets of Lagrangian results. This overshoot has been previously observed. ${ }^{19}$

From Figure 3 we see that the higher-order Godunov scheme better matches the density peak at $x \approx .75$, while the Lagrange plus remap scheme shows a small overshoot here. On the other hand the latter shows sharper resolution of the contact discontinuities at $x \approx .6$ and $x \approx .75$. The density between $x \approx .65$ and $x \approx .75$ also seems to be better represented by the Lagrange plus remap scheme. We note that the PPM results of Woodward and Colella ${ }^{1}$ show better resolution of the contact discontinuities than our Godunov results. The implementation of PPM used for those results includes a contact detection and steepening scheme. If that scheme had been disabled, those PPM results would have been essentially the same as the Godunov results shown here. ${ }^{28}$ Contact detection and steepening are not implemented in the present Godunov scheme because parabolic interpolation appears to be essential to its success; moreover, steepening appears to introduce spurious numerical artifacts in multidimensional calculations. ${ }^{28}$

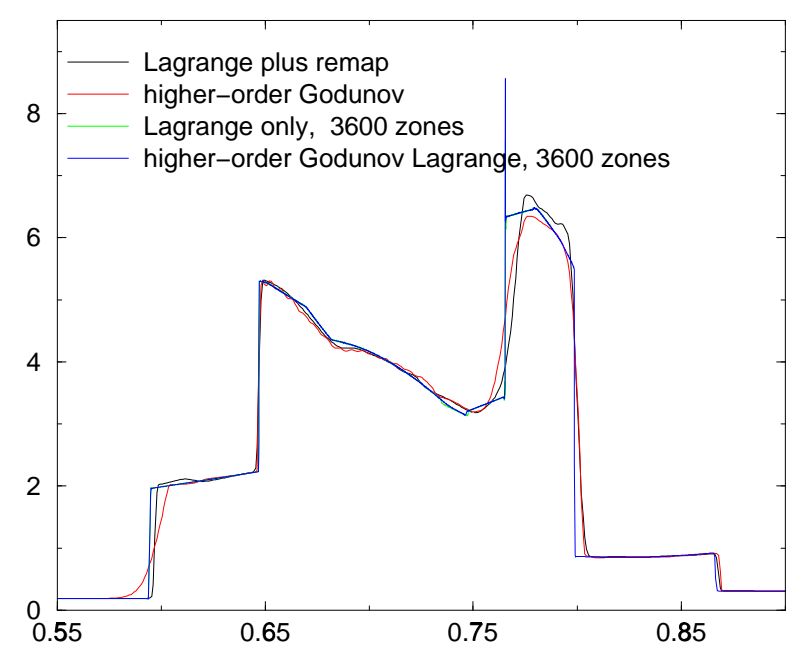

Fig. 3 Interacting blast wave problem at $t=$ .038. Closeup of wave interaction region.

We next examine the effect of advecting $E$ directly as opposed to advecting $e$ and $k e$ separately. (The former is the strategy used in BBC. ${ }^{1}$ ) Note that when $E$ is advected directly, the remapped value of $e$ is defined as the difference between $E$ and the kinetic energy found from the remapped velocity. In the upper plot of Figure 4 we see the results at $t=.038$ for three Lagrange plus remap calculations. The first density profile shows the same results displayed in Figure 3. The second profile plots the density when $E$ is advected directly. This profile contains a number of noticeable deficiencies. Woodward and Colella ${ }^{1}$ observed that these deficiencies were due to the computation of unphysically high densities at early time. They corrected the problem by using a minmod limiter in the advection step. The third profile show our results for this strategy. Note that these results are similar to those found with BBC by Woodward and Colella. ${ }^{1}$

In the lower half of Figure 4, we examine the early time behavior of the two energy remap approaches employing van Leer limiters. We indeed 
interacting blast wave: rho at $\mathrm{t}=0.03800$

1200 zones total on $[0,1]$
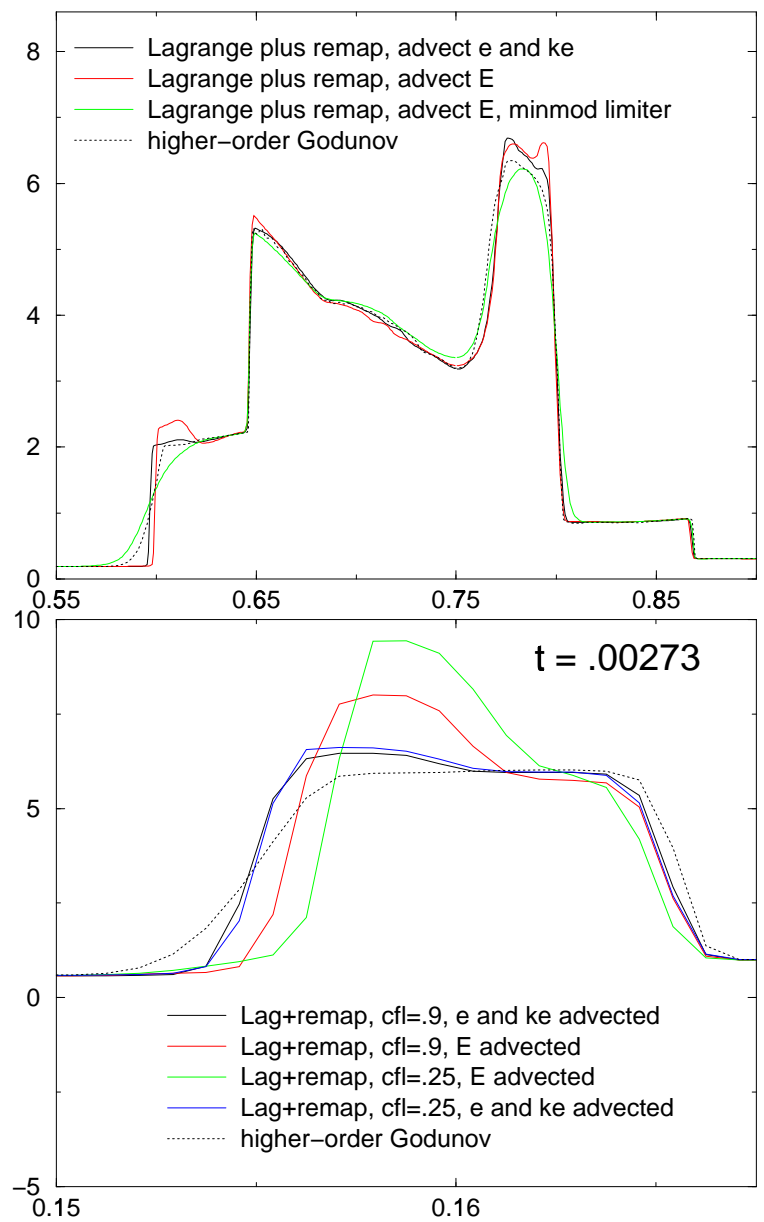

Fig. 4 Effect of energy advection strategies on solution of the interacting blast wave problem. Godunov results are shown for comparison.

see that when $E$ is advected directly, the density at early time is much higher than when $e$ and ke are remapped separately. Moreover, we see that the problem actually worsens as the CFL number is lowered. We conjecture that when $e$ and $k e$ are remapped separately, the early time overshoots are reduced because the effective total energy slopes are limited to a greater amount.

Double Mach reflection of a strong shock.

For this problem ${ }^{1}$ the flow domain has length 3.5 and width 1.0. At $t=0$ a Mach 10 planar shock in a $\gamma=1.4$ polytropic gas impinges at an angle of $60^{\circ}$ on the lower face of the domain at a distance of .3 from the left end of the domain. The preshock density and pressure are 1.4 and 1, respectively. The lower face of the domain is treated as a reflecting wall to the right of the impingement point; to the left, it is treated as an outflow boundary. At the top face inflow boundary conditions are imposed which match the exact evolution of the shock as it traverses the boundary. The other boundary conditions are inflow at the left and outflow at the right. The flow is self-similar and characterized as follows. The first reflected shock, the incident shock, and the first Mach stem meet at a triple point. The first contact discontinuity extends from this triple point down to the reflecting wall, at which point it is deflected into a wall jet that flows back towards the Mach stem. The contact discontinuity and wall jet exhibit small rollups induced by a Kelvin-Helmholtz instability. The wall jet also displays a larger rollup induced by the presence of the Mach stem. A curved reflected shock is connected to the first reflected shock. A second weaker Mach stem extends from this point to the first contact discontinuity. There is also a second slipline attached to this point which is too weak to appear in our computational results.

We compute the flow on a $1120 \times 320$ grid with the two methods. The density at $t=.21$ is displayed in Figure 5. The higher-order Godunov scheme is somewhat better at both suppressing oscillations in the postshock region of the left end of the curved reflected shock and not suppressing the small rollups in the wall jet. The results also show a smaller rollup of the wall jet just before the Mach stem. The Lagrange plus remap scheme, on the other, is better at suppressing oscillations in the middle and right sections of the postshock region of the curved reflected shock. Both schemes exhibit a spurious wave emanating where the Mach 10 shock intersects the top boundary. This wave, also observed by Woodward and Colella ${ }^{1}$ is due to the mismatch between the exact and the numerical representations of the shock outside and inside, respectively, the top boundary. The spurious wave has a slightly more deleterious effect on the Lagrange plus remap solution. The spurious contour in the higher-order Godunov results just outside the curved reflected shock at $x \approx 1.5, y \approx .5$ delimits a small perturbation that is also present in the Lagrange plus remap results but which does not appear in the plot with our choice of contour levels.

We next examine the effect of using a spatially operator split advection scheme that alternates $\xi \eta$ and $\eta \xi$ sweep patterns, each sweep taking a full pseudo-timestep, but which otherwise uses the advection algorithm described above. The density at $t=.21$ computed is displayed in Figure 6. We see that the Kelvin-Helmholtz instability along the wall jet is somewhat suppressed. The results otherwise do not differ significantly from those in Figure 5.

We finally examine the effect of using the standard 

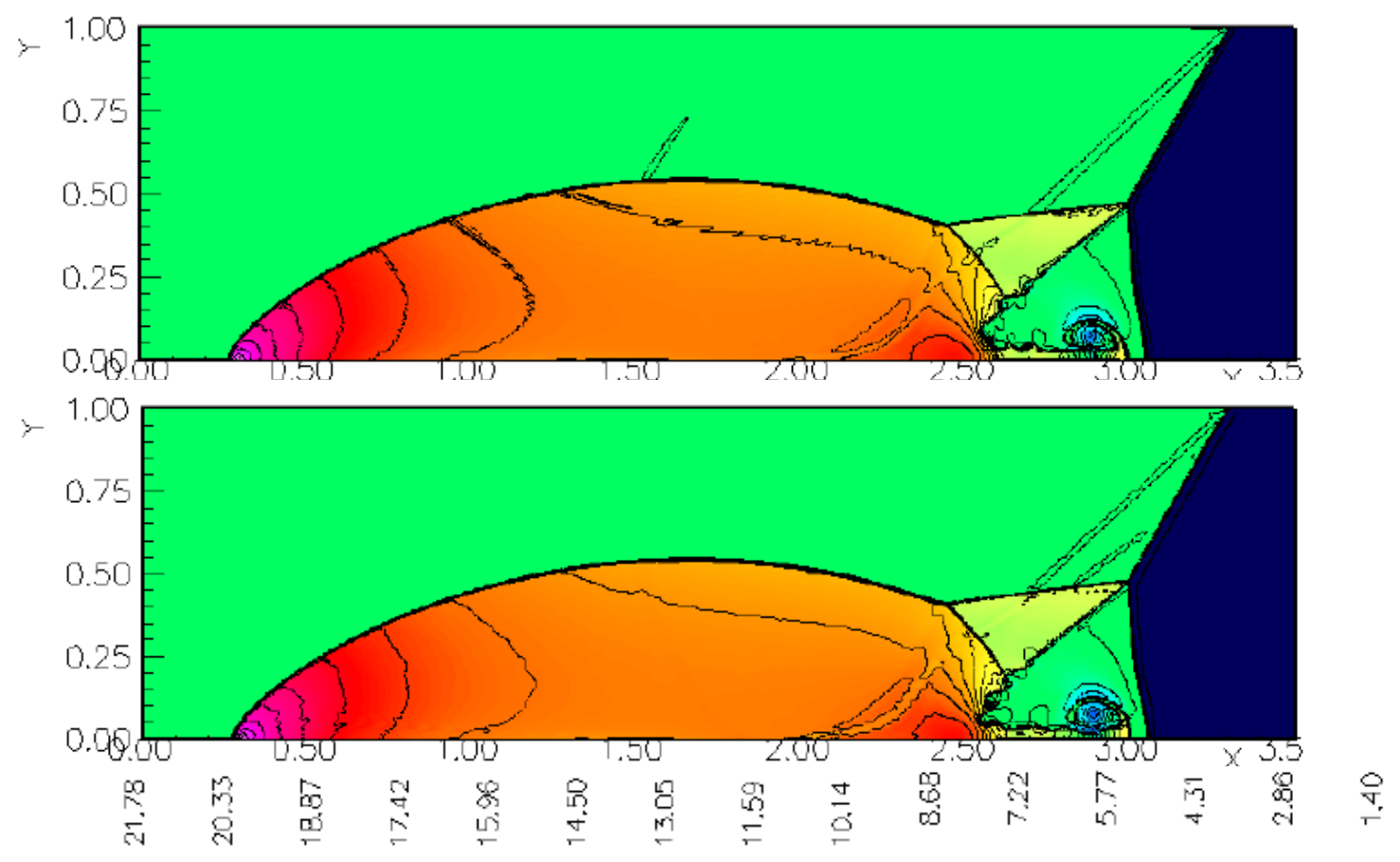

Fig. 5 Double Mach reflection of a Mach 10 shock. Thirty density contours are used in each plot. The density contours range from 1.4 to 22.51. Higher-order Godunov results are shown above Lagrange plus remap results. The same contour scale is used in Figures 6 and 7 .

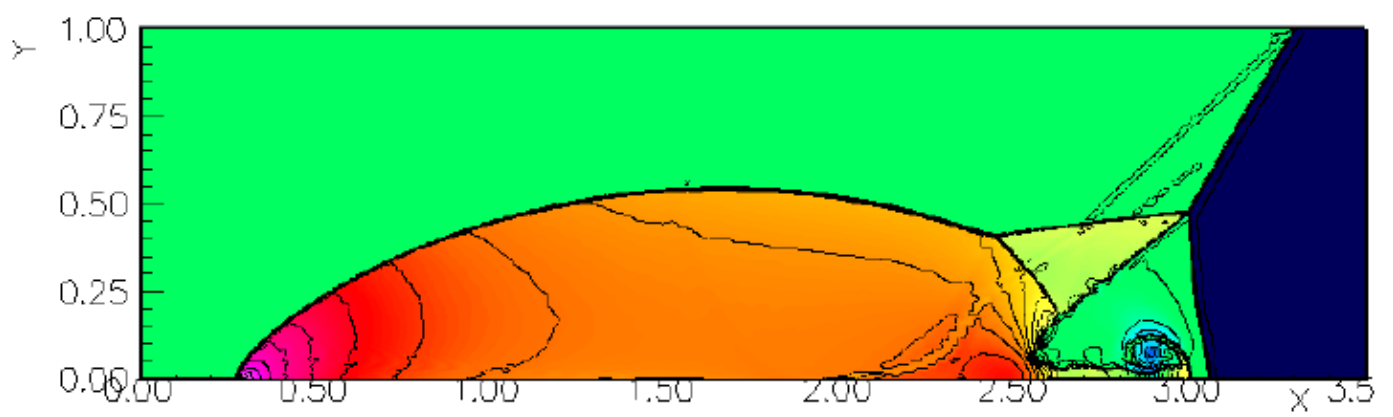

Fig. 6 Double Mach reflection computed by Lagrange plus remap scheme with spatially operator split advection scheme.

Lagrange viscosity. The computed density at $t=.21$ is shown in Figure 7. Using the standard Lagrange viscosity results in a poor representation of the Mach stem extending down from the kink in the reflected shock. This poor representation is also present in the $\mathrm{BBC}$ results reported by Woodward and Colella ${ }^{1}$ where it is attributed to the linear viscosity term in the standard Lagrange viscosity used by BBC. Otherwise, the results are again otherwise essentially the same as those in Figure 5.

\section{Oblique shock wave refraction.}

In this problem we model a shock $(M=1.89)$ in a $\gamma=1.4$ polytropic gas impinging on a den- sity interface at an angle of incidence of $58^{\circ}$. The preshock conditions in cgs units are $u=v=0$ and $p=1.01325 \times 10^{6}$. The preshock densities are $1.223 \times 10^{-3}$ upstream of the interface and $6.243 \times 10^{-3}$ downstream. The flow domain is 1.12 $\mathrm{cm}$ wide by $.72 \mathrm{~cm}$ high. The initial shock location is $.2 \mathrm{~cm}$ from the left boundary and the density interface intersects the lower boundary $.0975 \mathrm{~cm}$ further to the right. Reflecting wall boundary conditions are imposed at the upper and lower faces. The other boundary conditions are inflow at the left and outflow at the right.

This problem is a simplification of one in which 


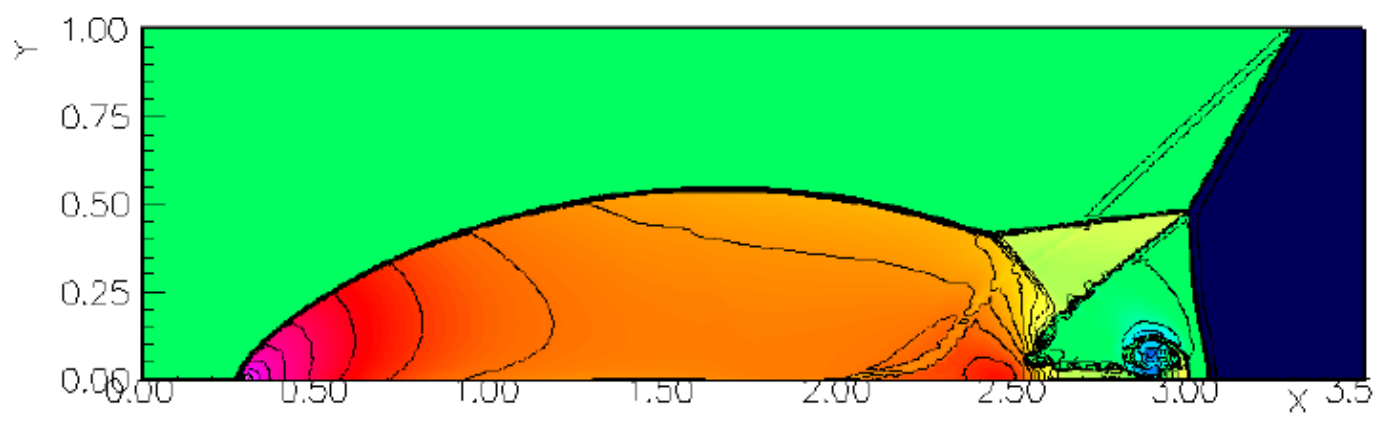

Fig. 7 Double Mach reflection computed by Lagrange plus remap scheme using the standard Lagrange viscosity.

a Mach 1.89 shock refracts at an air $/ \mathrm{SF}_{6}$ interface, ${ }^{29,30}$ the simplification being the treatment of both gases as polytropic with equal $\gamma$ 's of 1.4. The flow is characterized as follows. The incident shock, a Mach stem, a curved reflecting shock, and a weak contact discontinuity meet at a triple point. The contact extends from the triple point to the deflected density interface. Upstream of this intersection, the deflected density interface is characterized by rollups induced by a Kelvin-Helmholtz instability. A transmitted shock propagates into the denser fluid. The presence of the lower wall induces two additional flow features: the density interface undergoes rollup and the transmitted shock undergoes direct Mach reflection.

We compute the flow on a $896 \times 576$ grid with the two methods to $t=1.16667 \times 10^{-5}$. The density at that time in a subregion of the flow domain containing the direct Mach reflection at the lower wall is displayed in Figure 8. The results mainly differ along the density interface and near the incident shock triple point (which is outside the region displayed.) The Lagrange plus remap scheme computes a more damped rollup pattern and a slower growth rate of the shear layer than does the higher-order Godunov method. The damping can be seen especially in the upper right corner of the lower plot in Figure 8. The Lagrange plus remap algorithm computes a Mach stem height at the incident shock triple point of $.028 \mathrm{~cm}$ and an angle between the reflected shock and the Mach stem of $70^{\circ}$. The higher-order Godunov algorithm computes corresponding values of $.019 \mathrm{~cm}$ and $60^{\circ}$. Both codes, however, compute the same location of the incident shock Mach stem and the same angle between the Mach stem and the interface, namely, $78^{\circ}$.

\section{LeBlanc shock tube.}

In this shock tube problem ${ }^{22}$ the initial discontinuity separates a region of very high energy and density from one of low energy and density. The initial discontinuity is at $x=3 .(\rho, e, u)=(1, .1,0)$

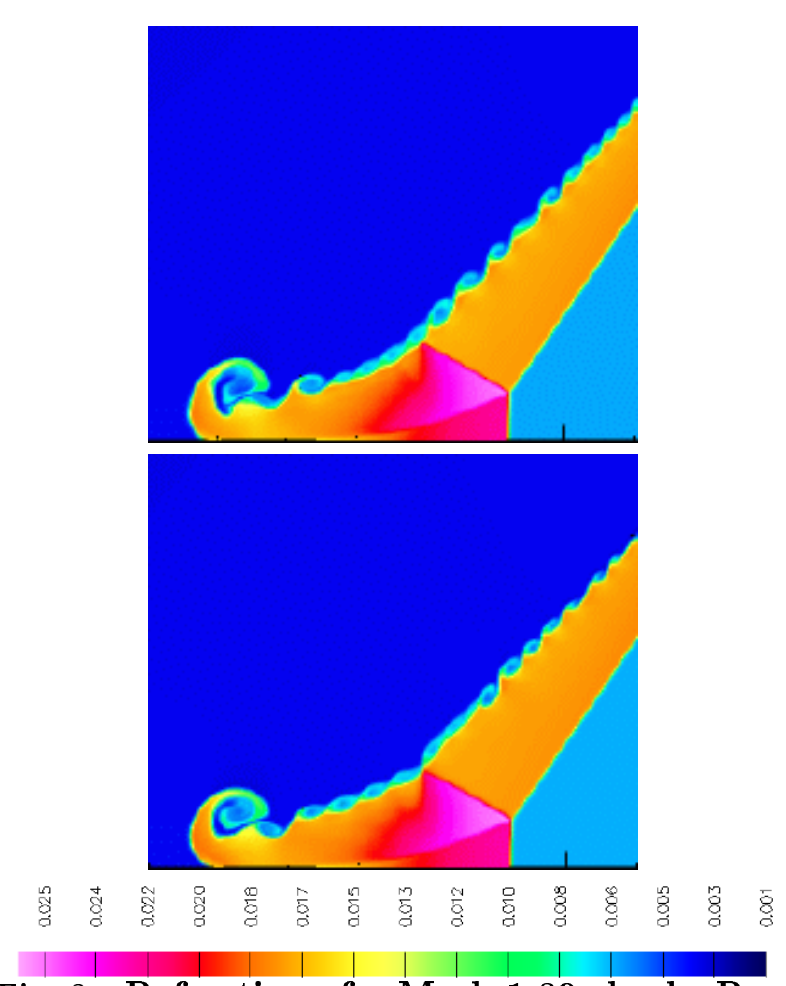

Fig. 8 Refraction of a Mach 1.89 shock. Region is a $.4 \mathrm{~cm}$ square centered $.6 \mathrm{~cm}$ from the left edge of the flow domain. The density in the entire flow domain ranges from $\mathbf{0 0 1 2 2 3}$ to $\mathbf{0 2 6 0 8 3}$. Higher-order Godunov results are shown above Lagrange plus remap results.

for $x<3$ and $\left(.001,10^{-7}, 0\right)$ for $x>3$. The gas is polytropic with $\gamma=5 / 3$. The solution consists of a strong rarefaction moving to the left, and a contact discontinuity and a shock moving to the right.

We compute the flow with both methods on grids of 180 and 1440 zones. For comparison, we also compute the flow both with a Lagrangian higherorder Godunov method ${ }^{27}$ and with the staggered grid Lagrange scheme on the same domain. We also compute the exact solution using an exact Riemann solver. The internal energies for all four methods 
and both grids as well the exact solution at $t=6$ are shown in Figure 9. On the 180 zone grid, all four methods have difficulty computing the position of the shock. The two Lagrangian methods best compute this position, while the two Eulerian scheme show roughly equal and opposite errors. Both Lagrangian methods, however, compute large overshoots in the postshock value. The higher-order Godunov method is best at computing the contact discontinuity, while the Lagrange plus remap scheme is more successful than the two Lagrangian methods. The two Eulerian methods both compute overshoots at the contact, the higher-order Godunov method the least, while the two Lagrangian methods show large oscillations there. On the 1440 zone grid, the higher-order Godunov results best match the exact solution, although they do underestimate the speed of the shock slightly and show a small undershoot at the contact discontinuity. The Lagrange plus remap scheme and the staggered grid Lagrange scheme both overestimate the speed of the shock. The Lagrange plus remap scheme computes a large but relatively narrow overshoot in the density at contact discontinuity. The staggered grid Lagrange scheme spreads this overshoot over a wider region. The higher-order Godunov Lagrange scheme shows the worst results. It overestimates the speed of the shock and spreads the overshoot at the contact over the entire region between the contact and the shock.

\section{Discussion and conclusions}

The results in the previous section demonstrate that a staggered grid, Lagrange plus remap, artificial viscosity scheme can compute results for Eulerian gas dynamics that are comparable to those of a cell centered, direct Eulerian, higher order Godunov method. Moreover, they show that certain features of the current Lagrange plus remap scheme contribute to its improved performance relative to the method examined by Woodward and Colella. ${ }^{1}$ Three critical features in particular are the use of the monotonic artificial viscosity in the Lagrange step and the use of a van Leer (or comparable) limiter in conjunction with the separate advection of internal and kinetic energies in the remap step.

The results for several of the test problems warrant additional discussion. The interacting blast wave results demonstrate that in some cases the Lagrange plus remap approach can resolve contact discontinuities much better than the higher-order Godunov approach. The double Mach reflection results demonstrate that the monotonic artificial viscosity contributes to the improved performance of the Lagrange plus remap scheme. They also suggest
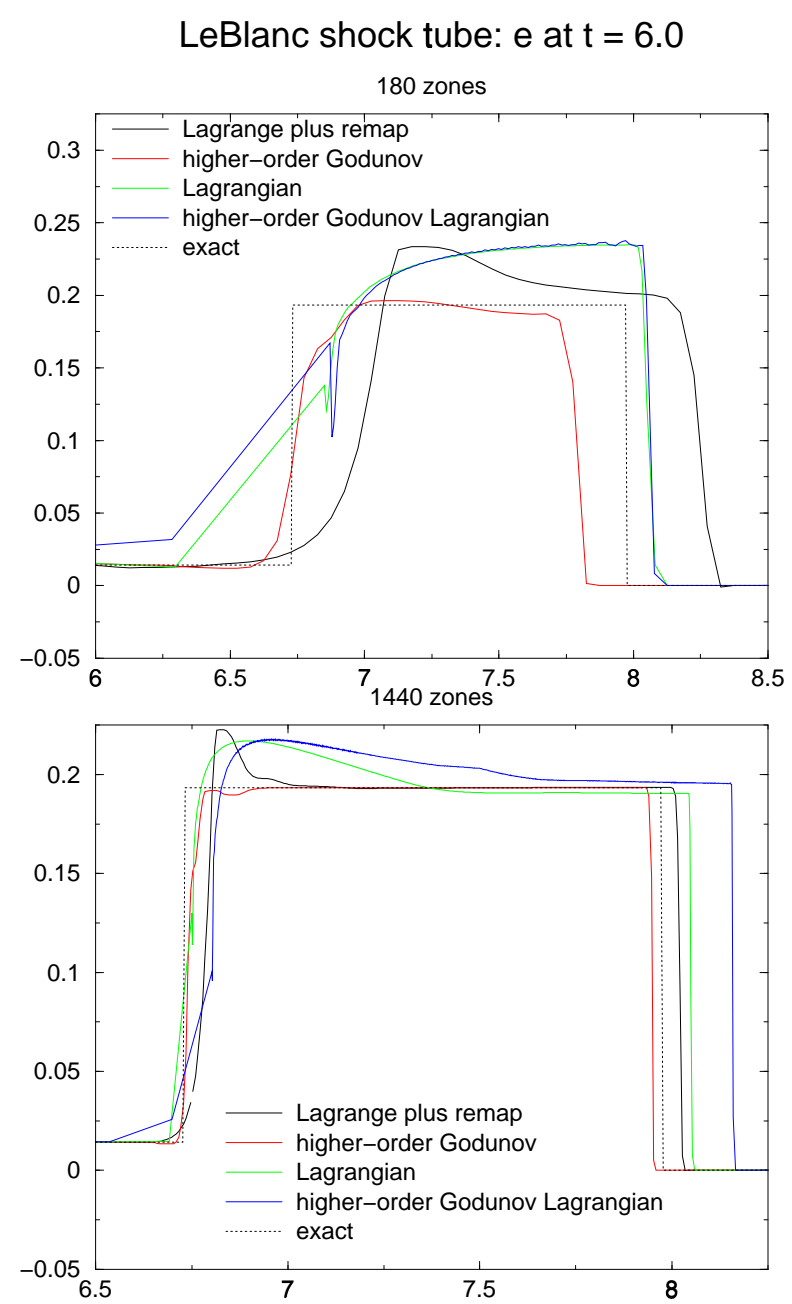

Fig. 9 Internal energy at $t=6$ for the LeBlanc shock tube on grids of 180 and 1440 zones. Closeup of the region surrounding the contact discontinuity and the shock.

that the unsplit nature of the corner transport upwind scheme contributes as well; however, this claim warrants further examination. The shock refraction results are inconclusive but do serve to show that the Lagrange plus remap scheme can compute the development of a Kelvin-Helmholtz instability along a shear interface. The LeBlanc problem is the one problem for which the Lagrange plus remap scheme did not fare as well as the higher-order Godunov method. The problems experienced by the Lagrange plus remap scheme, in particular the density overshoots at the contact discontinuity, do seem to originate in the Lagrange step. Moreover, these problems are independent of whether an artificial viscosity or a Godunov approach is used for shock capturing. Conversely, it is the direct Eulerian approach, and not the higher-order Godunov methodology itself, that enables the direct Eulerian Godunov scheme here to 
compute accurate results for the LeBlanc problem.

The work in this paper was done in conjunction with the development of a structured grid local adaptive mesh refinement scheme ${ }^{31}$ suitable for use with a staggered grid ALE methodology. Part of this work will entail implementation of the Lagrange plus remap algorithm in two-dimensional cylindrical coordinates as well as in three-dimensions. In future work, as that development proceeds, we will further our comparison of higher-order Godunov and staggered grid Lagrange plus remap schemes.

\section{Acknowledgments}

This work was performed under the auspices of the U.S. Department of Energy by University of California Lawrence Livermore National Laboratory under contract No. W-7405-Eng-48. The codes implementing the algorithms described in this paper were developed using SAMRAI, ${ }^{32}$ an object-oriented framework for the development of structured grid adaptive mesh refinement applications.

\section{References}

${ }^{1}$ Woodward, P. and Colella, P., "The Numerical Simulation of Two-Dimensional Fluid Flow with Strong Shocks," J. Comput. Phys., Vol. 54, 1984, pp. 115-173.

${ }^{2}$ Jameson, A., "Positive Schemes and Shock Modelling for Compressible Flows," Proceedings, 8th Finite Elements in Fluids Conference, Barcelona, September, 1993.

${ }^{3}$ Loh, C. and Liou, M., "A New Lagrangian Method for Three-Dimensional Steady Supersonic Flows," J. Comput. Phys., Vol. 113, 1994, pp. 224-248.

${ }^{4}$ Miller, G. and Puckett, E., "A High-Order Godunov Method for Multiple Condensed Phases," J. Comput. Phys., Vol. 128, 1996, pp. 134-164.

${ }^{5}$ Hirt, C. W., Amsden, A., and Cook, J., "An Arbitrary Lagrangian-Eulerian Computing Method for All Flow Speeds," J. Comput. Phys., Vol. 14, 1974, pp. 227-253.

${ }^{6}$ Benson, B. J., "Computational Methods in Lagrangian and Eulerian Hydrocodes," Comp. Meth. Appl. Mech. Eng., Vol. 99, 1992, pp. 235-394.

${ }^{7}$ Darlington, R., McAbee, T., and Rodrigue, G., "A Study of ALE Simulations of Rayleigh-Taylor Instability," Comp. Phys. Comm., Vol. 135, 2001, pp. 58-73.

${ }^{8}$ Smith, R., "AUSM(ALE): A Geometrically Conservative Arbitrary Lagrangian-Eulerian Flux Splitting Scheme," J. Comput. Phys., Vol. 150, 1999, pp. 268-286.

${ }^{9}$ Shestakov, A., Prasad, M., Milovich, J., Gentile, N. A., Painter, J., Furnish, G., and Dubois, P., "The ICF3D Code," Comp. Meth. Appl. Mech. Eng., Vol. 187, 2000, pp. 181-201.

${ }^{10}$ Tipton, R., Unpublished report, Lawrence Livermore National Laboratory, 1990.

${ }^{11}$ Wilkins, M. L., "Calculation of Elastic-Plastic Flow," Meth. Comp. Phys., Vol. 3, 1964, pp. 211-263.

${ }^{12}$ Wilkins, M. L., Computer Simulation of Dynamics Phenomena, Springer-Verlag, New York, 1999.

${ }^{13}$ Colella, P., "Multidimensional Upwind Methods for Hyperbolic Conservation Laws," J. Comput. Phys., Vol. 87, 1990, pp. 171-200.
${ }^{14}$ Bell, J., Colella, P., Trangenstein, J., and Welcome, M., "Adaptive Mesh Refinement on Moving Quadrilateral Grids," Proceedings, AIAA 9th Computational Fluid Dynamics Conference, Buffalo, New York, June 14-16, 1989, p.471-579.

${ }^{15}$ Jun, B., "A Simple Advection Scheme for Material Interface," Tech. Rep. UCRL-JC-139912, Lawrence Livermore National Laboratory, 2000.

${ }^{16}$ Benson, B. J., "An Efficient, Accurate, Simple ALE Method for Nonlinear Finite Element Programs," Comp. Meth. Appl. Mech. Eng., Vol. 72, 1989, pp. 205-350.

${ }^{17}$ Colella, P., Glaz, H., and Ferguson, R., "Multifluid Algorithms for Eulerian Finite Difference Methods," Unpublished, 1993.

${ }^{18}$ Pember, R., Greenough, J., and Colella, P., "An Adaptive, Higher-Order Godunov Method for Gas Dynamics in Three Dimensional Orthogonal Curvilinear Coordinates," Tech. Rep. UCRL-JC-123351, Lawrence Livermore National Laboratory, 1996.

${ }^{19}$ Christensen, R., "Godunov Methods on a Staggered Mesh - an Improved Artificial Viscosity," Tech. Rep. UCRLJC-105269, Lawrence Livermore National Laboratory, 1990.

${ }^{20}$ Noh, W., Unpublished report, Lawrence Livermore National Laboratory, 1956.

${ }^{21}$ Colella, P. and Woodward, P., "The Piecewise Parabolic Method (PPM) for Gas-Dynamical Simulations," J. Comput. Phys., Vol. 54, 1984, pp. 174-201.

${ }^{22}$ Benson, B. J., "Momentum Advection on a Staggered Mesh," J. Comput. Phys., Vol. 100, 1992, pp. 143-162.

${ }^{23}$ van Leer, B., "Towards the Ultimate Conservative Difference Scheme. V. A Second-Order Sequel to Godunov's Method," J. Comput. Phys., Vol. 32, 1979, pp. 101-136.

${ }^{24}$ Colella, P., "A Direct Eulerian MUSCL Scheme for Gas Dynamics," SIAM J. Sci. Stat. Comput., Vol. 6, 1985, pp. 104-117.

${ }^{25}$ Colella, P. and Woodward, P., "The Piecewise Parabolic Method (PPM) for Gas-Dynamical Simulations," J. Comput. Phys., Vol. 54, 1984, pp. 174-201.

${ }^{26}$ Colella, P. and Puckett, E., Finite Difference Methods for Computational Fluid Dynamic, Cambridge University Press, to be published. Also available as ftp://math.ucdavis.edu/pub/users/egp/

Colella_and_Puckett/.

${ }^{27}$ Saltzman, J. and Colella, P., "Second Order Corner Coupled Upwind Transport Methods for Lagrangian Hydrodynamics," Tech. Rep. LA-UR-85-678, Los Alamos National Laboratory, 1985.

${ }^{28}$ Colella, P., Private communication, 2000.

${ }^{29} \mathrm{Abd}-\mathrm{El}-\mathrm{Fattah}, \mathrm{A}$. and Henderson, L., "Shock waves at a fast-slow interface," J. Fluid. Mech., Vol. 86, 1978, pp. 15-32.

${ }^{30}$ Henderson, L. and Puckett, E., "Anomalous refraction of shock waves in materials with general equations of state. Part I. The shock pair system," Trans. Royal Soc. London A, submitted, 1993.

${ }^{31}$ Berger, M. and Colella, P., "Local Adaptive Mesh Refinement for Shock Hydrodynamics," J. Comput. Phys., Vol. 82, 1989, pp. 64-84.

${ }^{32}$ Hornung, R. and Kohn, S., "Future Directions for Adaptive Mesh Refinement in ASCI and other LLNL Simulation Projects," Tech. Rep. UCRL-ID-12833, Lawrence Livermore National Laboratory, 1997. 